\title{
Phronesis
}

\section{Interventions du superviseur lors de séances de rétroaction visant le développement de la réflexivité : étude de la variabilité inter-superviseurs Supervisor Interventions during retroactions: study of the variability between supervisors (inter-variability)}

\section{Marie Bocquillon, Arnaud Dehon et Antoine Derobertmasure}

Volume 4, numéro 1, hiver 2015

L’activité humaine au coeur du travail

URI : https://id.erudit.org/iderudit/1031201ar

DOI : https://doi.org/10.7202/1031201ar

Aller au sommaire du numéro

Éditeur(s)

Université de Sherbrooke

ISSN

1925-4873 (numérique)

Découvrir la revue

Citer cet article

Bocquillon, M., Dehon, A. \& Derobertmasure, A. (2015). Interventions du superviseur lors de séances de rétroaction visant le développement de la réflexivité : étude de la variabilité inter-superviseurs. Phronesis, 4(1), 14-27. https://doi.org/10.7202/1031201ar
Résumé de l'article

En formation initiale des enseignants, le développement de la réflexivité est souvent analysé en considérant le point de vue du futur enseignant. Or, le rôle du superviseur notamment lors d'échanges avec les futurs enseignants peut être primordial. Dans le cadre d'activités pédagogiques intégrées dans l'axe des savoir-faire de la formation, dix entretiens de rétroaction menés par deux superviseurs sont analysés afin d'appréhender d'éventuelles différences dans leurs interventions et d'éventuelles incidences sur le développement de la réflexivité des futurs enseignants. 


\title{
Interventions du superviseur lors de séances de rétroaction visant le développement de la réflexivité : étude de la variabilité inter-superviseurs
}

\author{
Marie BOCQUILLON*, Arnaud DEHON*, Antoine DEROBERTMASURE*
}

\author{
${ }^{*}$ Université de Mons \\ Institut d'Administration scolaire \\ 18, Place du Parc \\ Mons, Belgique \\ marie.bocquillon@umons.ac.be \\ arnaud.dehon@umons.ac.be \\ **Université de Mons \\ Cellule facultaire de pédagogie universitaire \\ 18, Place du Parc \\ Mons, Belgique \\ antoine.derobertmasure@umons.ac.be
}

Mots-clés : Superviseur, rétroaction, réflexivité, futurs enseignants

Résumé : En formation initiale des enseignants, le développement de la réflexivité est souvent analysé en considérant le point de vue du futur enseignant. Or, le rôle du superviseur notamment lors d'échanges avec les futurs enseignants peut être primordial. Dans le cadre d'activités pédagogiques intégrées dans l'axe des savoir-faire de la formation, dix entretiens de rétroaction menés par deux superviseurs sont analysés afin d'appréhender d'éventuelles différences dans leurs interventions et d'éventuelles incidences sur le développement de la réflexivité des futurs enseignants.

\section{Title : Supervisor Interventions during retroactions: study of the variability between supervisors (inter-variability)}

Keyword: Supervisor, retroaction, reflexivity, future teachers

Abstract: In initial teacher training, the studies on reflexive practice are often considered on the future teacher point of view. Nevertheless, the action of a supervisor is very important during an activity of retroaction. In one of the core activities, the know-how, ten interviews are analyzed to determine the differences between the practice of two supervisors and their impact in terms of reflective practice of the future teachers. 


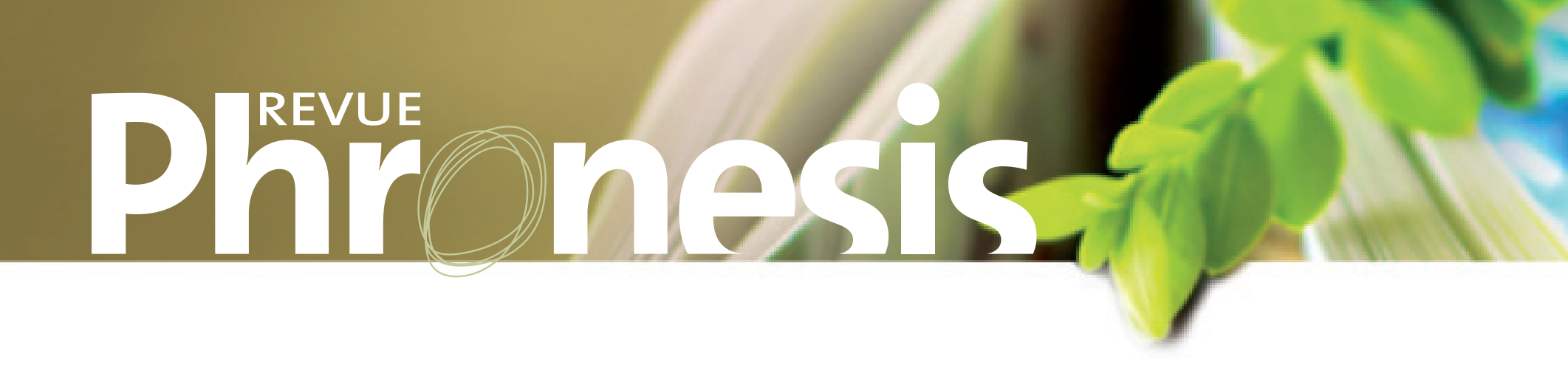

\section{Introduction}

La formation initiale des enseignants du secondaire supérieur (niveau lycée en France) en Belgique francophone est depuis 2001 (D.08-022001) définie par un décret mettant en évidence 13 compétences à développer chez les futurs enseignants. Ces compétences sont centrées sur le développement de la réflexivité, de l'identité professionnelle et de la professionnalité. Si dans le décret, les axes et contenus de formation sont précisés, les universités, opérateurs de formation, gardent un certain degré de liberté quant aux activités de formation. Ainsi, pour l'axe des savoir-faire, l'Institut d'Administration scolaire de l'Université de Mons a mis en place, pour la formation des agrégés de l'enseignement secondaire supérieur de la Faculté de Psychologie et des Sciences de l'Éducation et la Faculté Warocqué d'Économie-Gestion, un dispositif (Derobertmasure \& Dehon, 2012) s'articulant autour de séances permettant de "planifier, gérer et évaluer des situations d'apprentissage " (compétence 12 du référentiel) et de "porter un regard réflexif sur sa pratique et organiser sa formation continuée " (compétence 13 du référentiel). Concrètement, cela se traduit par une alternance entre activités à l'université ( $40 \mathrm{~h}$ de septembre à décembre) et stages en milieu professionnel (60 h de janvier à mai). Ces activités sont les mêmes pour les futurs enseignants des deux facultés et sont réparties sur une année académique. Les séances à l'université se composent notamment d'une étape de microenseignement, d'une étape d'autoscopie vidéo et d'une étape de rétroaction entre le futur enseignant et un superviseur, activités pédagogiques retenues pour leur qualité de développement de la pratique réflexive (Hatton et Smith, 1995).

Si la réflexivité fait l'objet de nombreuses recherches portant sur sa modélisation (e.g. Jorro, 2005; Kolb, 1984; Korthagen, 2001; Mc Alpine, Weston, Beauchamp, Wiseman \& Beauchamp, 1999; Schön, 1994; Sparks-Langer, Simmons, Pasch, Colton \& Starko, 1990; Van Manen, 1977; Zeichner \& Liston, 1996) et sur sa manifestation auprès de futurs enseignants (e.g. Sparks-Langer \& al., 1990; Hatton \& Smith, 1995; Jorro, 2005; De Cock, 2007; Derobertmasure, 2012), la question du développement de celle-ci en contexte de formation initiale soulève encore plusieurs interrogations telles que : comment objectiver les traces de réflexivité des futurs enseignants et comment les évaluer? En effet, même si les dispositifs de formation se veulent professionnalisants, il est difficile d'évaluer l'impact de ce type de dispositif à l'aide d'indicateurs fiables (Derobertmasure, Dehon \& Demeuse, 2009). De même, plusieurs auteurs ont mis en évidence le fait que la notion de réflexivité a été conceptualisée de différentes manières, ce qui la rend difficile à opérationnaliser et à évaluer (Beauchamp, 2006 ; Collin, 2010 ; Correa Molina, Collin, Chaubet \& Gervais, 2010 ; De Cock, 2007 ; Derobertmasure, 2012 ; Hatton \& Smith, 1995 ; Saussez, Ewen \& Girard, 2001). En outre, la place et l'intervention du superviseur lors des séances de rétroaction ont été peu investiguées. Pourtant, considérant l'interaction verbale comme un des moteurs de la réflexivité (Correa Molina \& al., 2010), le rôle du superviseur semble primordial pour contribuer au développement de la réflexivité du futur enseignant. En effet, un entretien de supervision bien mené est une occasion de développement professionnel et permet au futur enseignant de développer sa réflexivité (Correa Molina, 2008). Cet article tente donc d'apporter des éléments de réponse à cette double problématique : d'une part, l'objectivation et l'évaluation des traces de réflexivité des futurs enseignants et, d'autre part, la caractérisation d'interventions des superviseurs.

L'intervention du superviseur est étudiée dans un contexte de séances de rétroaction durant lesquelles le futur enseignant et un superviseur échangent à partir de l'enregistrement vidéo de la première leçon du futur enseignant (réalisée en microenseignement, c'est-à-dire lorsque le futur enseignant s'exerce à donner cours devant ses collègues). Avant cette séance de rétroaction, le futur enseignant, en autoscopie (individuellement), sélectionne deux scènes de 5 minutes sur les 40 minutes de sa prestation. Ces deux scènes sont à la base des échanges entre le superviseur et le futur enseignant. Durant les rétroactions, le superviseur, comme le futur enseignant, est libre de prendre la parole et d'interrompre le visionnage des deux scènes.

Sur la base de modèles théoriques opérationnalisés (Derobertmasure, 2012; Derobertmasure \& Dehon, 2012), les propos des superviseurs sont étudiés pour appréhender la variabilité inter-superviseurs entre les deux superviseurs se répartissant les séances de rétroaction. En effet, il semble essentiel dans l'évaluation du dispositif d'étudier la variabilité des interventions des superviseurs dont la pratique doit permettre à tous les étudiants d'atteindre les mêmes objectifs.

Cet article présente les principaux résultats de l'analyse des propos des superviseurs, ainsi que certains résultats liés aux propos des futurs enseignants. Au préalable, le cadre théorique ayant guidé cette analyse et la méthodologie sont présentés. 
Dans cette section, les trois modèles théoriques et les indicateurs quantitatifs utilisés pour analyser les propos des superviseurs et des futurs enseignants sont présentés. Le premier modèle est centré sur le superviseur et aborde les fonctions de ses propos. Le second, le modèle de la pratique d'enseignement-apprentissage (Derobertmasure et Dehon, 2012), catégorise les objets de la pratique d'enseignement-apprentissage pouvant être évoqués durant les séances de rétroaction aussi bien par le futur enseignant que par le superviseur. Le troisième liste un ensemble de processus réflexifs pouvant être mobilisés par les futurs enseignants durant ces séances (Derobertmasure, 2012). Ce dernier modèle est utilisé afin de déterminer, au moins en partie, des liens entre les interventions des superviseurs et les manifestations de la réflexivité des futurs enseignants.

\subsection{Les fonctions du superviseur}

La plupart des travaux menés sur la réflexivité se sont principalement focalisés sur le futur enseignant et ont, par conséquent, moins investigué le rôle du superviseur, pourtant primordial (Correa Molina \& al., 2010; Derobertmasure, 2012). Dans le but de mieux appréhender les séances de rétroaction en se concentrant sur le superviseur, ses interventions sont définies. Ces éléments vont amener à construire, d'une part, une grille permettant d'analyser les propos du superviseur et d'autre part de comparer les interventions des deux superviseurs (variabilité inter-superviseurs).

À partir des travaux de Blanchet (1989), Berthier (2010) et De Lièvre (2005), Derobertmasure (2012) identifie cinq fonctions dans les interventions du superviseur : (1) la fonction évaluative, (2) la fonction pédagogique, (3) les questions liées au futur enseignant, (4) la fonction sociale et (5) la fonction structurante. La fonction évaluative permet au superviseur d'aider le futur enseignant à positionner sa pratique par rapport aux objectifs qu'il doit atteindre. Cette fonction comprend les rétroactions et approbations du superviseur. La fonction pédagogique intègre les interventions du superviseur liées aux compétences méthodologiques et disciplinaires permettant d'aider le futur enseignant à porter un regard réflexif. La troisième fonction - les questions liées au futur enseignant - recouvre les propos portant sur le futur enseignant dans sa personne. La fonction sociale reprend les interventions conduisant à rassurer le futur enseignant lors de séances de rétroaction, parfois porteuses de stress. Enfin, la fonction structurante, se définit comme "la clarification et l'interprétation de la situation ou des propos, le recentrage des propos, la réitération et le fait de compléter les propos du futur enseignant » (Derobertmasure, 2012, p. 244).

À partir de ce premier modèle, ces cinq fonctions ont été enrichies par les travaux de Correa Molina (2005a; 2005 b; 2008), Korthagen (2001; 2011), Korthagen et Kessels (1999), Korthagen et Vasalos (2005) afin d'opérationnaliser ces fonctions et permettre, in fine, d'affiner l'analyse des propos du superviseur.

La fonction évaluative (1) se divise en deux sous-catégories qui sont la rétroaction stéréotypée et la rétroaction spécifique (De Landsheere \& Bayer, 1974). La rétroaction stéréotypée a comme fonction de montrer au futur enseignant que le superviseur suit bien la conversation ( $O k »$ ) et le second, la rétroaction spécifique, est davantage constructif et porte sur un élément de la pratique enseignante ( Si je voulais mettre encore en évidence, c'est que tu les raccroches vraiment à leur réalité, à leur formation. On imagine que c'est un public de secondaire, leur rôle d'agent d'éducation... Dans les autres leçons que j'ai pu déjà observer, on est souvent dans la matière»).

Dix sous-catégories composent la fonction pédagogique (2). On y retrouve :

- (1) la transmission d’un savoir («Théorie avec un T majuscule») («C'est ce que certains pédagogues préconisent, c'est de partir d’une situation-problème. [...]»;

- (2) la transmission d'un avis, d'un conseil («théorie avec un t minuscule ») (« De toute façon, l'erreur fait partie de l'apprentissage, parce que si on ne faisait aucune erreur, on n'aurait pas grand-chose à apprendre. Et l'erreur donne des renseignements sur la façon dont on se représente les choses, ça permet de réajuster pour qu'elle soit correcte »);

- (3) la transmission visant le décryptage des pratiques ("Ça, je dirais, c’est une réponse qui apparaît un peu par magie. La réponse apparaît parce qu'elle la connaît »);

- (4) l'invitation à poursuivre la phrase («C'est-à-dire?»);

- (5) la question qui encourage la justification («Et pourquoi alors l'actualité, vous y faites référence à ce moment-là et pas plus tôt?»);

- (6) la question liée à la situation observée, («Est-ce que c’est pas encore Damien qui répond?»);

- (7) la question métacognitive («Comment aurais-tu pu faire autrement pour quand même partir d'éléments concrets et réels?»);

- (8) la question visant à vérifier que l'étudiant suit la conversation («Tu vois?»);

- (9) la question de justification de la scène (« Pourquoi avoir choisi alors cette deuxième scène? »);

- (10) les consignes, objectifs de l’activité/de la formation («Ici, le but, c'est le développement professionnel et réfléchir ensemble à une pratique »).

La question liée au futur enseignant (3) et la fonction sociale (4) ne sont pas subdivisées. La première reprend les questions liées aux caractéristiques du futur enseignant, au stress lié à la présence de caméras ("Tu as peur des caméras? Tu es stressée? »)... La deuxième concerne les interventions par lesquelles le superviseur rassure le futur enseignant, dédramatise la situation, instaure un climat de confiance («Ce n'est pas une erreur, mais on commet tous cette formulation »). 
Enfin, la fonction structurante (5) est subdivisée en trois sous-catégories : compléter les propos de l'étudiant (« ... et il ne doit pas gérer les mauvaises réponses »), recentrer les propos («Alors, c’est quoi ta deuxième scène? ») et réitérer/refléter ("Ah oui, là ils demandent »).

\subsection{Les objets de la pratique d'enseignement-apprentissage (ou objets de la réflexivité)}

Collin (2010) catégorise les modèles de la réflexivité selon qu'ils soient à dominante (1) du type séquentiel, c'est-à-dire que la réflexivité est envisagée selon une série d'étapes (2) du type évaluatif, où les formes de réflexivité sont organisées hiérarchiquement, et (3) du type thématique, selon les contenus de la réflexivité. Le modèle de la pratique d'enseignement-apprentissage correspond davantage à un modèle de type thématique, car il permet de catégoriser le «ce sur quoi » portent la réflexivité des futurs enseignants et les propos des superviseurs. Dans la recherche présentée, les objets de la pratique d'enseignement-apprentissage se définissent au sens du microsystème (Perrenoud, 2001 in De Cock, 2007), c'est-à-dire du contexte de la classe. Le modèle retenu (Derobertmasure, 2012, p. 28) intègre plusieurs paramètres ou caractéristiques de la pratique d'enseignement-apprentissage. Il se compose de quatre pôles, se définissant comme les entités, physiques ou non, présentes dans une situation : l'enseignant, l'(es) élève (s), l'objet d'apprentissage et les résultats ou les produits de cet apprentissage. La notion de dispositif " qui est la manifestation observable des interactions et des relations entre quatre pôles » (Derobertmasure \& Dehon, 2012, p. 28) y est centrale. Les pôles - élève, enseignant, objet et résultats/produits - sont intégrés au dispositif à travers quatre processus qui sont respectivement : (1) le processus d'implication, (2) le processus d'organisation, (3) le processus de transposition et (4) le processus évaluatif'. La relation entre le dispositif et les processus est bidirectionnelle puisqu'elle explique l'intégration des pôles dans le dispositif, mais permet également de comprendre l'influence du dispositif sur les pôles.

\section{Domaine des contenus}

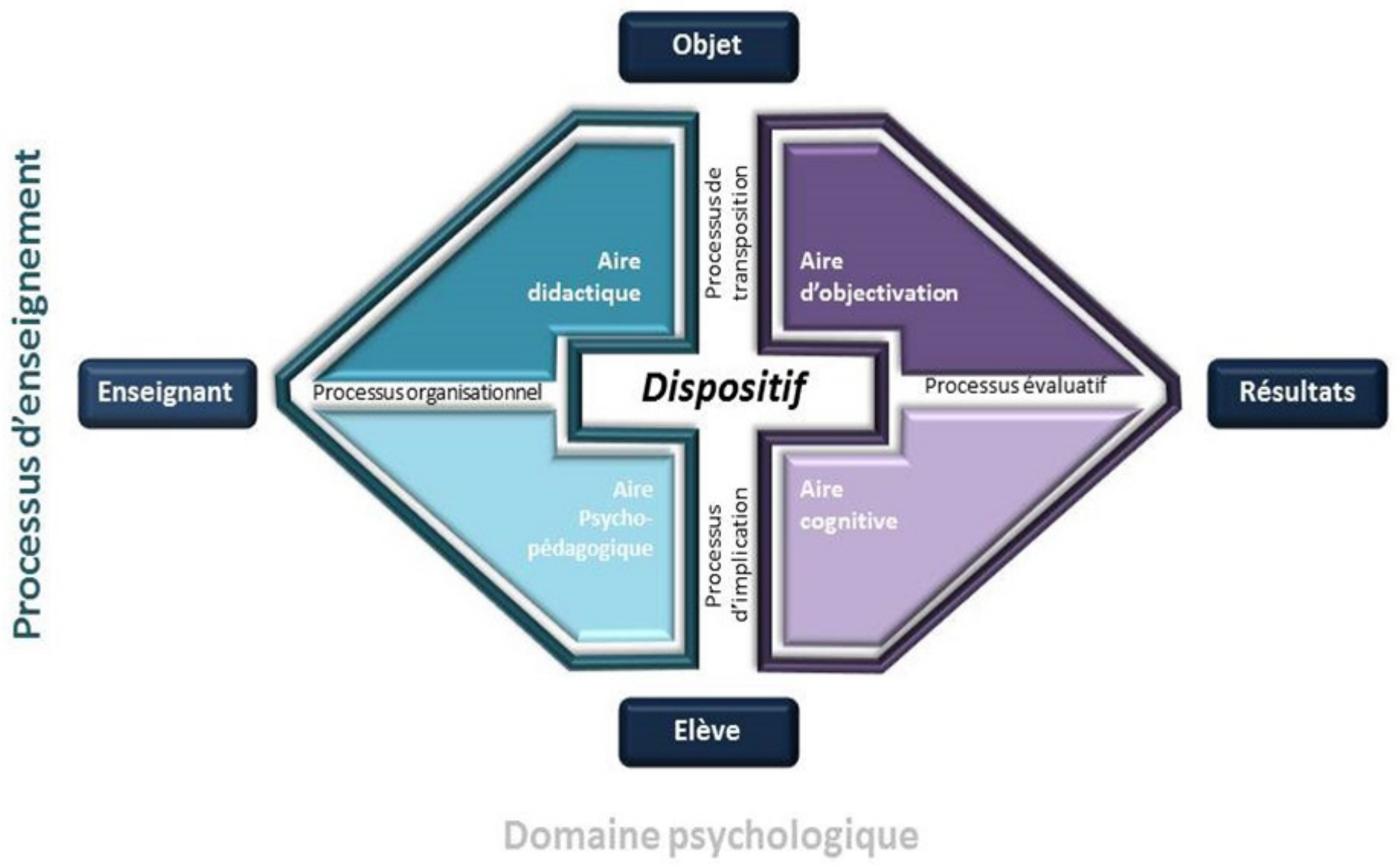

Figure 1 : modèle de la pratique d'enseignement-apprentissage

Si les processus définissent les relations entre les pôles et le dispositif, il existe au croisement de ces processus, ces pôles et du dispositif, des 1 Depuis la version précédente du modèle (Derobertmasure et Dehon, 2012), l’appellation des processus a été modifiée théoriquement afin de mieux préciser ce qu'ils
recouvrent : le processus transposition s'intitulait « processus d'institutionnalisation » et le processus évaluatif « régulation ». 
espaces pouvant définir la nature des interventions ou des relations entre ces éléments. Ainsi, au croisement des pôles enseignant et objet, et des processus organisationnel et de transposition, on peut définir un espace, une aire, dont la nature est didactique, car il intègre les relations entre l'enseignant et l'objet d'apprentissage, et se compose des processus organisationnel et de transposition. Pratiquement, cette aire se caractérise par les interventions liées au contenu d'enseignement par les choix opérés, le discours énoncé...

Quatre aires sont ainsi définies au sein du modèle : (1) l'aire didactique, (2) l'aire d'objectivation qui met en relation l'objet et les résultats ou produits d'apprentissage et les processus de transposition et de régulation, (3) l'aire cognitive ${ }^{2}$ entre les pôles résultats et élève et les processus de régulation et d'implication et enfin (4) l'aire psychopédagogique définissant les interventions entre les pôles élève et enseignant et les processus d'implication et organisationnel.

Au niveau de l'analyse des propos, la version adaptée du modèle permet de catégoriser et de déterminer à quels objets le discours, ou une partie de celui-ci, fait référence. Cependant, les quatre aires ont été définies plus précisément en fonction de plusieurs sous-catégories et de façon à correspondre aux objectifs de la recherche, à savoir caractériser les propos des futurs enseignants et des superviseurs.

L'aire didactique se rapporte aux éléments relatifs au contenu et à la façon de le préparer, le transposer, le transmettre et le présenter. Elle permet de cibler, dans les propos, ce qui a trait au choix et à l'organisation des contenus, au discours didactique, à la préparation de leçon ou au support didactique. L'aire psychopédagogique concerne les aspects relationnels, la gestion temporelle et spatiale, les activités proposées, la dynamique insufflée, les interactions verbales ou écrites... On y distingue les actes de langage, les activités sur les contenus, le climatespace, la dynamique corporelle, la gestion de l'apprentissage, l'adéquation à la préparation de leçon et le rythme-temps. L'aire cognitive, liée à l'élève dans ses aspects cognitifs, ses connaissances préalables et son profil d'apprentissage, renvoie aux sous-catégories suivantes : l'apprentissage/raisonnement/réflexion/motivation de l'élève, le niveau de l'élève/profil de l'élève. Enfin, l'aire d'objectivation, qui rassemble les manifestations verbales et non verbales des élèves dans la construction de l'objet d'apprentissage, comprend les actions des élèves et la manifestation de la compréhension/réponses de l'élève.

À ces catégories permettant d'appréhender les objets de la pratique d'enseignement-apprentissage, une catégorie « contexte de formation » a été ajoutée pour permettre le codage des éléments faisant référence au contexte, c'est-à-dire les références à la séance de microenseignement, aux avis remis par les autres futurs enseignants prenant la place « d'élèves » lors de ces séances et, au statut de stagiaire/remplaçant. L'ajout de cette catégorie illustre la démarche mixte/itérative mise en place (Mukamurera, Lacourse \& Couturier, 2006). La démarche est donc à la fois déductive/délibérative, puisque le cadre théorique est utilisé afin de guider l’analyse (Savoie-Zajc, 2000; 2004, in Mukamurera \& al., 2006); et inductive, puisque les grilles de codage préétablies sont enrichies « d’autres éléments qui émergent des données " (Mukamurera \& al., p. 114).

\subsection{Les processus réflexifs du futur enseignant}

Sur la base de différents modèles séquentiels et évaluatifs (Fenstermacher, 1996; Hatton \& Smith, 1995; Hensler, Garant \& Dumoulin, 2001; Jorro, 2005; Kolb, 1984; Schön, 1994; Sparks-Langer \& al., 1990; Van Manen, 1977), un modèle de la réflexivité a été élaboré (Derobertmasure et Dehon, 2012, pp. 30-31) et permet de mettre en évidence treize processus réflexifs répartis selon trois niveaux.

Le premier niveau fait appel aux processus qui sont indispensables à une pratique réflexive, car préliminaires à toute prise de recul. Il s'agit des processus permettant de faire état de la pratique :

- narrer/décrire sa pratique («Je demande d'analyser et de synthétiser le graphique »);

- questionner (" Je suis en pleine réflexion sur les textes lacunaires en fait, parce que je ne sais pas pourquoi moi je suis partie làdedans avec je sais pas les cours qu'on a eus");

- prendre conscience («Donc aussi par rapport à la matière, il faut qu'on se rende compte que c'est peut-être difficile pour eux»);

- pointer ses difficultés/ses problèmes («Parce que bon malgré tout, préparer des cours, ce n’est pas facile et c’est vrai qu'expliquer les matières, ce n'est pas facile»).

Le deuxième niveau rassemble les processus faisant référence à une norme, explicite ou implicite, ou un modèle ou une intention. Ces processus permettent une prise de distance par rapport à la pratique. Six processus définissent le deuxième niveau :

- (5) légitimer sa pratique selon une préférence ("Je trouve que dans le secondaire supérieur, si les élèves, si c'est une classe qui d'emblée aime participer, désigner quelqu'un... je ne vois pas trop l'intérêt. Je préfére demander qui veut y aller tout simplement »);

- (6) légitimer sa pratique en fonction d'arguments contextuels ("J'essaye de, en plus bon cette année pour mes cinquièmesixième, ce sont de chouettes classes, donc je peux faire ce genre de choses. L’année passée, ça aurait été plus difficile, ils étaient 28, pas spécialement motivés, donc il y a aussi le contexte de la classe qui joue beaucoup ");

- (7) légitimer sa pratique en fonction d’arguments pédagogiques ou éthiques («Oui, je crois que travailler individuellement, c’est bien. Mais, travailler en groupe aussi, c'est bien, parce que c'est toujours nécessaire d'avoir une autonomie, mais aussi de savoir travailler en groupe. Même en privé, c'est comme ça, on ne peut pas travailler individuellement du début à la fin »);

- (8) intentionnaliser sa pratique («Et vu que le but, c'était quand même traiter eux-mêmes l'information [...] »;

- (9) évaluer sa pratique ("Je suis pas assez... Enfin où je ne communique pas assez avec eux, je ne suis pas assez en interaction »);

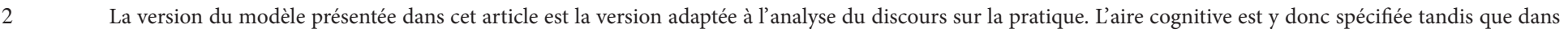

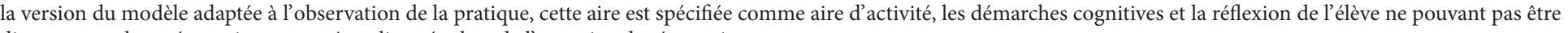
directement observées mais pouvant être discutées lors de l'entretien de rétroaction. 
- (10) diagnostiquer ("Je me dis dans une classe d'élèves, je n’aurais pas fait pareil et puis, il y aurait eu des questions et puis ça aurait été des élèves. Voilà, ces deux facteurs-là m’ont fait abréger »).

Enfin, trois processus forment le troisième niveau et se définissent comme les processus tournés vers une expérience future, qu'elle soit hypothétique ou concrète :

- (11) proposer une ou des alternatives à sa pratique ("Là, j’aurais dû demander à un autre élève de compléter si ce n’était pas complet, plutôt qu'à chaque fois compléter moi-même»);

- (12) explorer une ou des alternatives à sa pratique ("I'aurais dî quand même la [la vidéo] repasser et couper en extraits, à chaque fois après l'extrait demander à plusieurs élèves leurs réponses pour mettre tout en commun et avoir une réponse vraiment complète»);

- (13) théoriser («Donc, il faut traduire le langage savant en langage accessible, dans un langage accessible aux étudiants [...]»).

Si le modèle se structure selon trois niveaux, il faut considérer la notion de niveau différemment qu'au sens purement hiérarchique (Van Manen, 1977; Hatton \& Smith, 1995). En effet, chaque niveau a son importance et il ne s'agit pas de présenter le niveau le plus « haut » comme l’objectif ultime du développement de la réflexivité : par exemple, la réflexion technique est essentielle (Van Manen, 1977; Hatton \& Smith, 1995) ou encore le premier niveau défini par Van Manen permet de calmer les préoccupations de survie personnelle des enseignants novices (Beckers, 2009). Il semble même que ce ne soit pas le niveau atteint qui indique le degré de qualité de la réflexion, mais le nombre de niveaux que l'enseignant est capable de mobiliser (Collin, 2010).

\subsection{Indicateurs quantitatifs}

Quatre indicateurs quantitatifs sont utilisés : (1) le temps de passation de chaque entretien, (2) le nombre de mots par futur enseignant et (3) par superviseur, ainsi que (4) l'initiative de la parole (Derobertmasure, 2012).

Le nombre de mots prononcés par chacun des deux locuteurs est pris en compte, car si l'étudiant parle moins que le superviseur, cela peut indiquer un approfondissement réflexif moindre (Boutet et Gagné, 2009).

L'initiative de la parole est également intéressante, car comme le soulignent Boutet et Gagné (2009), l'origine du sujet de conversation est un signe de l'approfondissement réflexif : plus celle-ci est dans le camp du futur enseignant, plus elle constitue le signe d'un approfondissement réflexif. Les interventions peuvent être (1) proactives, (2) réactives ou (3) interactives (Derobertmasure, 2012) lorsque respectivement (1) il y a prise de parole spontanée, (2) il y a prise de parole en retour d'une intervention proactive ou (3) toutes autres interventions. Il est à remarquer que ce classement concerne les interventions portant sur un même objet de la pratique d'enseignement-apprentissage. Si pendant des interventions interactives, l'une des deux personnes aiguille le discours sur un autre objet, on parlera d'intervention proactive menant à une intervention réactive.

\section{Méthodologie}

\section{$2.1 \quad$ Échantillon}

Deux superviseurs constituent notre échantillon. Ils occupent à la fois le rôle de superviseur et celui de chercheur puisque leur objet d'étude, à savoir l'analyse des traces de réflexivité et des gestes professionnels, porte sur le dispositif de formation mis en place et dans lequel ils interviennent. Lors de l'année 2011 - 2012, ils possédaient des profils très proches (diplomation, fonction occupée au sein de l'institution de formation - assistant et assistant de recherche - , thématique de recherche, âge...).

Douze entretiens au total ont été passés lors de cette année académique. Pour chaque superviseur, cinq entretiens ont été retenus. Un enregistrement pour le superviseur 1 étant de qualité médiocre et ne permettant pas d'analyser précisément les échanges a été retiré de l'échantillon. Afin de garder le même nombre d'entretiens pour chaque superviseur, à savoir cinq, un entretien du superviseur 2 a été aléatoirement retiré et ce, en respectant l'équilibre du nombre de futurs enseignants issus de chacune des deux facultés pour chaque superviseur. Les entretiens retenus pour chaque superviseur sont au nombre de trois étudiants pour la faculté 1 (FWEG) et deux pour la faculté 2 (FPSE).

\subsection{L'analyse de contenu et le logiciel NVivo}

L'analyse de contenu, bien qu'elle soit mobilisée dans de nombreux travaux, est difficile à définir de façon exhaustive et consensuelle (Derobertmasure \& Robertson, 2013). On peut considérer que l'analyse de contenu d'un document consiste à "rechercher les informations qui s'y trouvent, dégager le sens ou les sens de ce qui est présenté, formuler et classer tout ce que "contient" ce document » (Mucchielli, 2006, p. 24) en limitant le recours à l'intuition et à la subjectivité (Derobertmasure \& Robertson, 2013).

Parmi plusieurs types d'analyses de contenu, c'est l'analyse de contenu thématique qui a été choisie dans le cadre de cette étude. Il s'agit de coder le contenu d'un corpus fragment par fragment (Fallery \& Rodhain, 2007). Le chercheur doit donc associer un code à chaque unité du texte en fonction de son contenu. 
Le logiciel $\mathrm{NVivo}^{\circ}$ a été choisi, car il est fréquemment utilisé pour les analyses thématiques et de manière générale dans les recherches en sciences humaines, ce qui semble lui conférer une certaine validité (Derobertmasure \& Robertson, 2013). La retranscription des dix entretiens a également été effectuée à l'aide du logiciel $\mathrm{NVivo}^{\circ}$. Lors de l'analyse de contenu, chaque retranscription a été découpée en unités de codage, c'est-à-dire le "segment de contenu à considérer comme unité de base en vue de la catégorisation et du comptage fréquentiel " (Bardin, 2001, p. 135). Le chercheur peut se baser sur un critère sémantique (basé sur le sens) ou sur un critère syntaxique (mot, phrase, paragraphe ou message) pour déterminer son unité de codage (Derobertmasure \& Robertson, 2013). Dans le cadre de cette étude, c’est le critère sémantique qui a été choisi pour déterminer les unités de codage, car il permet plus de flexibilité (Quintin, 2007), d'autant plus que le critère syntaxique n'est pas pertinent, dans ce cas-ci, car les entretiens n'ont pas été retranscrits par les émetteurs du message oral, mais par un retranscriveur.

\subsection{Validation de la procédure de codage}

En plus de la définition opérationnelle des catégories et sous-catégories, la procédure de codage a fait l'objet d'une procédure de validation par contrôle de la fiabilité inter-codeurs, sur la base de la formule de Miles et Huberman (2003, p. 126) : nombre d'accords entre les codages/ (nombre d'accords + nombre de désaccords entre les codages).

Dans ce cas-ci, 30 unités de codage ont été codées par un second chercheur en ce qui concerne les processus réflexifs et 30 unités en ce qui concerne les interventions du superviseur. Dans un premier temps, les taux d'accords s'élevaient à $90 \%$ pour les processus, ce qui correspond à un taux satisfaisant, car supérieur à $80 \%$, et à $70 \%$ pour les fonctions du superviseur. Après ajustement et une réexplicitation des catégories des fonctions du superviseur, un nouveau score de $97 \%$ a été calculé, soit un taux satisfaisant.

Dans le cadre d'une autre recherche (Bocquillon, Derobertmasure, Demeuse, Kozlowski \& Artus, 2014), en suivant la même procédure, mais en se basant sur 50 unités codées, les objets de la situation d'enseignement-apprentissage ont révélé des accords de $78 \%$ dans un premier temps et de $98 \%$ dans un second.

\subsection{Remarques méthodologiques concernant l'analyse des données}

Pour effectuer ces analyses, le nombre de mots plutôt que d'autres indicateurs tels que le nombre d'unités de codage a été privilégié. En effet, le nombre de mots prononcés pour chaque catégorie n’est pas influencé par les opérations de codage. Cette remarque prend tout son sens pour l'analyse des propos proactifs, réactifs et interactifs. L'exemple suivant permet de mieux comprendre le choix méthodologique adopté. Il s'agit d'une intervention proactive d'un futur enseignant qui porte sur plusieurs objets de la pratique d'enseignement-apprentissage (le discours didactique, le rythme-temps et l'apprentissage de l'élève).

«Ouais, parce que c'est trop oral et à partir d’un moment, je vais commencer à écrire au tableau [discours didactique], mais ça me prend beaucoup de temps moi-même d'écrire la réponse [rythme-temps], tandis que si l'élève va lui-même déjà, il retient aussi, ça le marque aussi le fait d'écrire lui-même [apprentissage de l'élève].»

En termes de codage, trois unités de codage ont été déterminées et codées dans la catégorie "étudiant proactif » : la première dans la souscatégorie " étudiant proactif, discours didactique ", la seconde dans " étudiant proactif, rythme-temps " et la dernière dans " étudiant proactif, apprentissage de l'élève ". Lors de l'analyse des résultats, le recours au nombre de mots codés dans chaque catégorie a donc été préféré afin d'éviter de "surestimer " le nombre d'interventions proactives (mais aussi réactives ou interactives), car le logiciel NVivo ${ }^{\circ}$ compte trois interventions proactives dans le cas de cet exemple, alors qu'il n'y en a qu' une, mais qui porte sur trois objets.

Le recours au nombre de mots comporte d'autres avantages en comparaison au recours à la durée de l'entretien : en plus d'être calculé de façon précise par le logiciel NVivo, il n'est pas influencé par le débit de parole des superviseurs et des futurs enseignants, ni par l'utilisation qu'ils font de la vidéo ("play», «pause», avance rapide) (Derobertmasure, 2012).

Néanmoins, que l'on ait recours au nombre de mots ou au nombre d'unités, une des limites de l'analyse thématique réside dans le fait qu'elle est basée sur la fréquence de certains thèmes dans le discours du sujet. Or, un thème fréquemment abordé n’est pas forcément important pour le sujet. Il est peut-être plus facilement verbalisable. À l'inverse, un thème peu présent dans le discours peut être important pour le sujet, mais réprimé ou difficile à mettre en mots (Ghiglione, Beauvois, Chabrol \& Trognon, 1980). Conscients de cette limite, nous avons tout de même eu recours au nombre de mots codés dans chaque catégorie afin d'objectiver les différents objets de la pratique d'enseignementapprentissage, les processus réflexifs et les fonctions présents dans les discours des sujets.

\section{Résultats}

Pour caractériser les interventions des superviseurs et des futurs enseignants, différentes analyses ont été effectuées à partir des modèles théoriques retenus : (1) le calcul du nombre de mots prononcés par chaque superviseur; (2) l'analyse des fonctions utilisées par les superviseurs; (3) l'analyse des objets de la pratique d'enseignement-apprentissage abordés par les superviseurs; (4) l'analyse de la proactivitéréactivité-interactivité des propos relatifs aux différents objets de la pratique d'enseignement-apprentissage; (5) l'analyse des processus 
réflexifs mobilisés par les futurs enseignants.

\subsection{Prises de parole des superviseurs}

Le premier point sur lequel se base la comparaison inter-superviseurs porte sur le nombre de mots moyen par rétroaction prononcés par chaque superviseur, car c'est un indicateur de l'opportunité laissée aux futurs enseignants de s'exprimer et donc d'approfondir leur raisonnement réflexif (Boutet \& Gagné, 2009). Le superviseur 1 prononce en moyenne 3291,6 mots par rétroaction $(\sigma=862,19$; Coefficient de Variation $=0,26)$ pour une durée moyenne de 45 minutes $(\sigma=4,94 ; \mathrm{CV}=0,11)$ alors que le superviseur 2 obtient une moyenne de 7006,4 mots par rétroaction $(\sigma=2427,38 ; \mathrm{CV}=0,35)$ pour une durée moyenne de 63 minutes $(\sigma=18,17 ; \mathrm{CV}=0,29)$. Malgré cette différence de prise de parole des superviseurs, on ne constate pas de différences majeures dans la prise de paroles des futurs enseignants. En effet, les futurs enseignants associés au superviseur 1 expriment en moyenne 3131,8 mots par entretien $(\sigma=1070,92$; CV $=0,34)$ et les futurs enseignants associés au superviseur 2 en prononcent en moyenne 3049,8 $(\sigma=924,68 ; \mathrm{CV}=0,30)$. L'observation du rapport moyen entre le nombre de mots prononcés par le futur enseignant et le superviseur donne respectivement : 1,05 pour le superviseur 1 et 2,30 pour le superviseur 2.

\subsection{Répartition des fonctions abordées par les superviseurs}

À partir du nombre total de mots prononcés par chaque superviseur lors des cinq entretiens ( $\mathrm{S} 1=16548$ mots et S2 = 35032 mots), leurs interventions sont comparées sur la base des fonctions mobilisées.

Tableau 1 : fonctions abordées par les superviseurs

\begin{tabular}{l|c|c} 
& Superviseur 1 & Superviseur 2 \\
\hline Consignes, objectifs & $14,26 \%$ & $2,26 \%$ \\
\hline Invitation à poursuivre & $0,02 \%$ & $0,01 \%$ \\
\hline Question justification, scène & $0,00 \%$ & $0,37 \%$ \\
\hline Question liée à la situation & $9,16 \%$ & $2,89 \%$ \\
\hline Question métacognitive & $3,69 \%$ & $1,43 \%$ \\
\hline Question qui encourage la justification & $0,30 \%$ & $0,28 \%$ \\
\hline Transmission & $58,25 \%$ & $76,46 \%$ \\
\hline Vérifier que le futur enseignant suit & $0,05 \%$ & $0,35 \%$ \\
\hline Autres & $0,45 \%$ & $0,36 \%$ \\
\hline Fonction évaluative -Rétroaction & $6,85 \%$ & $7,94 \%$ \\
\hline Phrase incomplète & $0,35 \%$ & $0,40 \%$ \\
\hline Question liée au futur enseignant & $0,28 \%$ & $0,35 \%$ \\
\hline Rassurer (fonction sociale) & $3,50 \%$ & $2,62 \%$ \\
\hline Structurer & $2,83 \%$ & $4,29 \%$ \\
\hline & $100,00 \%$ & $100,00 \%$
\end{tabular}

L'intervention la plus utilisée (en termes de volume de mots prononcés) par les superviseurs est la transmission. Elle est davantage utilisée par le superviseur 2 (76,46 \%) que par le superviseur 1 (58,25\%). Le superviseur 2 a donc un style davantage « transmissif » que le superviseur 1, qui pose davantage de questions liées à la situation $(9,16 \%$ contre $2,89 \%$ chez le superviseur 2$)$ et de questions métacognitives $(3,69 \%$ contre $1,43 \%$ chez le superviseur 2). Le superviseur 1 parle aussi davantage des consignes et des objectifs de l'activité (ou de la formation en général) $(14,26 \%$ contre $2,26 \%$ chez le superviseur 2$)$.

Les interventions de nature évaluative sont utilisées dans des proportions semblables par les deux superviseurs (6,85\% et 7,94 \%). Ensuite, les interventions de nature sociale (rassurer, dédramatiser) occupent 3,50\% des propos du superviseur 1 et 2,62 \% des propos du superviseur 2 . Enfin, les interventions de nature structurante occupent 2,83\% des propos du superviseur 1 et 4,29\% des propos du superviseur 2.

Les autres interventions (invitation à poursuivre, question de justification de la scène, question qui encourage la justification, vérifier que l'étudiant suit, question liée au futur enseignant, phrase incomplète, autres) occupent chacune moins de $1 \%$ des mots prononcés par les superviseurs. La catégorie « question de justification de la scène » est absente des propos du superviseur 1. Cela s'explique par le fait, en début d'entretien (lors de la présentation des consignes), que celui-ci explique généralement aux étudiants qu'ils devront justifier le choix de leurs scènes, mais ne leur redemande pas par la suite de manière explicite. Par contre, le superviseur 2 prévoit un moment lors de chaque entretien (à l'exception d'un entretien) où il demande aux futurs enseignants de justifier la scène qu'ils ont choisie de commenter. 
Le choix méthodologique adopté (recourir au nombre de mots prononcés) trouve ici une limite, car certains résultats sont rendus marginaux, car ils renvoient à peu de mots. Par exemple, lorsqu'on a recours au nombre de mots, la catégorie "vérifier que l'étudiant suit ", qui comprend des phrases très courtes telles que « Tu vois? », est considérée comme quasiment absente des propos des deux superviseurs $(0,05 \%$ des mots prononcés par le superviseur 1 et $0,35 \%$ des mots prononcés par le superviseur 2). Or, si au lieu de se baser sur le nombre de mots, on se base sur le nombre d'unités de sens codées dans la catégorie en question, soit $0,53 \%^{3}$ des unités de codage chez le superviseur 1 et 2,77 \% des unités de codage chez le superviseur 2, on constate que le superviseur 2 utilise régulièrement des interventions pour vérifier que l'étudiant suit (ex. : « Tu vois?»). Ce résultat peut être mis en relation avec le fait que ce superviseur transmet davantage que le superviseur 1. En effet, lorsqu' il transmet un savoir ou un conseil, le superviseur 2 ponctue son discours de petites phrases telles que « Tu vois? » afin de s'assurer que le futur enseignant est attentif. Pour les autres catégories (la transmission, les questions liées à la situation et les questions métacognitives), le recours aux unités de sens, bien qu'il fournisse quelques précisions - le discours transmissif serait moins direct que les deux autres catégories -, ne fournit pas de résultats marquants.

Étant donné que la transmission est l'intervention la plus utilisée par les deux superviseurs, il est opportun de s'intéresser plus en détail aux différents types de transmission. Les données présentées ont été obtenues en considérant que les propos d'un superviseur codés dans la catégorie « Transmission" valent $100 \%(\mathrm{~N}=9587$ mots pour le superviseur 1; $\mathrm{N}=26787$ mots pour le superviseur 2). Les deux superviseurs transmettent principalement de la théorie " avec un $t$ minuscule », c'est-à-dire de la théorie adaptée aux problèmes concrets rencontrés par le futur enseignant. Le superviseur 2 en transmet davantage ( $76,28 \%$ des mots codés dans la catégorie "Transmission ») que le superviseur 1 $(67,85 \%$ des mots codés dans la catégorie "Transmission »). La transmission de type « décryptage des pratiques » (qui consiste à mettre en évidence certains éléments de la situation dont le futur enseignant ne prend pas conscience) arrive en seconde position avec $31,41 \%$ des propos transmissifs du superviseur 1 et $22,42 \%$ des propos transmissifs du superviseur 2. Enfin, la "Théorie avec un T majuscule ", qui correspond à de la théorie «brute " est quasiment absente des propos transmissifs des deux superviseurs $(0,74 \%$ pour le superviseur 1 et $1,30 \%$ pour le superviseur 2 ).

En ce qui concerne les interventions de nature évaluative, en considérant que les propos d'un superviseur codés dans la catégorie «Feedbacks " valent $100 \%(\mathrm{~N}=1128$ pour le superviseur $1 ; \mathrm{N}=2780$ pour le superviseur 2$)$, on constate que les rétroactions constructives occupent $71,28 \%$ des mots du superviseur 1 et 72,55 \% de ceux du superviseur 2. Les rétroactions stéréotypées, quant à eux, occupent $28,72 \%$ des mots du superviseur 1 codés dans la catégorie «Feed-back » et 27,45\% de ceux du superviseur 2. À nouveau, si l'on recourt au nombre d'unités de codage de la catégorie "Feed-back ", on constate que, pour les deux superviseurs, il y a plus de rétroactions stéréotypées que de rétroactions constructives. Ce résultat n'est cependant pas « visible » en recourant au nombre de mots, car les rétroactions constructives comportent, par nature, plus de mots que les rétroactions stéréotypées. Néanmoins, quelle que soit la méthode choisie, on ne constate pas de variabilité inter-superviseurs en ce qui concerne les interventions de nature évaluative.

\subsection{Répartition des propos en fonction des objets de la pratique d'enseignement-apprentissage}

Si l'on se concentre sur les objets abordés dans les prises de parole des superviseurs, on remarque certaines similitudes et des proportions relativement semblables entre les superviseurs. Les résultats suivants sont calculés à partir du nombre total de mots prononcés par chaque superviseur (lors de ses cinq entretiens de rétroaction) au sujet des objets de la pratique d'enseignement-apprentissage, c'est-à-dire 13161 mots pour le superviseur 1 et 32150 mots pour le superviseur 2. L'aire psychopédagogique est la plus souvent commentée pour les deux superviseurs $(\mathrm{S} 1=57,02 \%$ des mots prononcés et $\mathrm{S} 2=59,65 \%)$. Viennent, ensuite, les autres aires du modèle qui présentent peu de différence : l'aire didactique avec $10,13 \%$ des propos du superviseur 1 et 12,44\% des propos du superviseur 2; l'aire cognitive est présente dans $15,59 \%$ des propos du superviseur 1 et $11,19 \%$ des propos du superviseur 2 et l'aire d'objectivation occupe 9,71 \% des propos du superviseur 1 et 10,06 \% des propos du superviseur 2. Concernant les pôles du modèle, ceux-ci sont globalement peu commentés : le pôle enseignant occupe $2,52 \%$ des propos du superviseur 1 et 2,05\% des propos du superviseur 2; le pôle objet est présent dans moins de $2 \%$ des propos de chacun des superviseurs; le pôle résultat n'est pas abordé par le superviseur 1 et très peu par le superviseur $2(0,36 \%)$; le pôle élève n'est commenté par aucun des superviseurs. Enfin, le contexte de formation est abordé dans 3,70\% des propos du superviseur 1 et dans $2,68 \%$ des propos du superviseur 2 .

Si globalement, les deux superviseurs semblent aborder les aires et les pôles selon une hiérarchie et dans des proportions proches, la focalisation sur les composantes de chacune des aires va permettre de mettre en évidence certaines distinctions tant sur les objets que sur la façon de les aborder (proactif, réactif ou interactif).

L'analyse de l'aire didactique ( $\mathrm{N}=1333$ mots pour le superviseur 1; $\mathrm{N}=4001$ mots pour le superviseur 2) montre que le superviseur 2 parle deux fois plus de la préparation de leçon ( $41,66 \%$ des mots prononcés relatifs à l'aire didactique) que le superviseur 1 (21,23\%). Il commente également plus le choix et l'organisation des contenus (22,34\% contre $15,90 \%$ chez le superviseur 1$)$. De son côté, le superviseur 1 mentionne plus le support didactique $(27,91 \%$ contre $14,62 \%$ chez le superviseur 2$)$ et le discours didactique $(34,96 \%$ contre $21,37 \%$ chez le superviseur 2).

En ce qui concerne l'aire psychopédagogique ( $\mathrm{N}=7504$ mots pour le superviseur 1; N= 19178 mots pour le superviseur 2), la différence la plus marquante entre les deux superviseurs concerne le climat-espace, qui occupe 17,53\% des mots prononcés par le superviseur 2 contre 
seulement $0,21 \%$ chez le superviseur 1 . De son côté, le superviseur 1 parle davantage de la gestion de l'apprentissage ( $57,68 \%$ contre $37,08 \%$ chez le superviseur 2). La deuxième sous-catégorie la plus commentée (les activités sur les contenus) est commentée dans des proportions similaires par les deux superviseurs (30,44 \% pour le superviseur 1 et $27,91 \%$ pour le superviseur 2 ). Les autres sous-catégories sont moins commentées : le rythme-temps (7,37 \% des propos du superviseur 1 et 11,09\% des propos du superviseur 2); la référence à la préparation de leçon (1,47 \% pour le superviseur 1 et 3,82 \% pour le superviseur 2$)$; la dynamique corporelle (2,36 \% pour le superviseur 1 et $0,83 \%$ pour le superviseur 2 ) et les actes de langage ( $0,48 \%$ pour le superviseur 1 et $1,74 \%$ pour le superviseur 2$)$.

Au niveau de l'aire cognitive ( $N=2052$ mots pour le superviseur 1; $N=3596$ mots pour le superviseur 2), les deux superviseurs font davantage référence au niveau et au profil des élèves (58,19\% des mots relatifs à l'aire cognitive du superviseur 1 et $67,41 \%$ de ceux du superviseur 2 ) qu'à leur apprentissage et à leur motivation (41,81\% des mots relatifs à l'aire cognitive du superviseur 1 et 32,59\% de ceux du superviseur 2). Pour ce qui est de l'aire d'objectivation ( $\mathrm{N}=1278$ mots pour le superviseur 1; $\mathrm{N}=3234$ mots pour le superviseur 2), les deux superviseurs parlent davantage de la manifestation de la compréhension et des réponses des élèves $(74,73 \%$ des mots relatifs à l'aire d'objectivation du superviseur 1 et 70,50\% de ceux du superviseur 2) que des actions des élèves (25,27\% des mots relatifs à l'aire d'objectivation du superviseur 1 et $29,50 \%$ de ceux du superviseur 2 ).

Tableau 2 : répartition des propos des superviseurs en fonction des objets de la pratique d'enseignement-apprentissage

\begin{tabular}{|c|c|c|c|c|c|}
\hline & A. psychopédagogique & A. didactique & \multicolumn{2}{|c|}{ A. cognitive } & A. d'objectivation \\
\hline Superviseur $1(\mathrm{~N}=13161)$ & $57,02 \%$ & $10,13 \%$ & \multicolumn{2}{|c|}{$15,59 \%$} & $9,71 \%$ \\
\hline \multirow[t]{3}{*}{ Superviseur $2(\mathrm{~N}=32150)$} & $59,65 \%$ & $12,44 \%$ & \multicolumn{2}{|c|}{$11,19 \%$} & $10,06 \%$ \\
\hline & \multicolumn{5}{|c|}{ Aire psychopédagogique } \\
\hline & \multicolumn{3}{|c|}{$\begin{array}{l}\text { Aire psychopédagog } \\
\text { Gestion de l'apprentissage }\end{array}$} & \multicolumn{2}{|c|}{ Activités sur les contenus } \\
\hline Superviseur $1(\mathrm{~N}=7504)$ & \multicolumn{3}{|c|}{$57,68 \%$} & \multicolumn{2}{|c|}{$30,44 \%$} \\
\hline \multirow[t]{3}{*}{ Superviseur $2(\mathrm{~N}=19178)$} & \multicolumn{3}{|c|}{$37,08 \%$} & \multicolumn{2}{|c|}{$27,91 \%$} \\
\hline & \multicolumn{5}{|c|}{ Aire didactique } \\
\hline & Préparation de leçon & \multicolumn{2}{|c|}{$\begin{array}{c}\text { Choix/organisation des } \\
\text { contenus }\end{array}$} & $\begin{array}{c}\text { Support } \\
\text { didactique }\end{array}$ & $\begin{array}{c}\text { Discours } \\
\text { didactique }\end{array}$ \\
\hline Superviseur $1(\mathrm{~N}=1333)$ & $21,23 \%$ & \multicolumn{2}{|l|}{$15,90 \%$} & $27,91 \%$ & $34,96 \%$ \\
\hline \multirow[t]{3}{*}{ Superviseur $2(\mathrm{~N}=4001)$} & $41,66 \%$ & \multicolumn{2}{|l|}{$22,34 \%$} & $14,62 \%$ & $21,37 \%$ \\
\hline & \multicolumn{5}{|c|}{ Aire cognitive } \\
\hline & \multicolumn{2}{|c|}{ Niveau et profil des élèves } & \multicolumn{3}{|c|}{ Apprentissage et motivation } \\
\hline Superviseur $1(\mathrm{~N}=2052)$ & \multicolumn{2}{|c|}{$58,19 \%$} & \multicolumn{3}{|c|}{$41,81 \%$} \\
\hline \multirow[t]{3}{*}{ Superviseur $2(\mathrm{~N}=3596)$} & \multicolumn{2}{|c|}{$67,41 \%$} & \multicolumn{3}{|c|}{$32,59 \%$} \\
\hline & & Aire d'obje & vatic & & \\
\hline & Compréhension et rép & nses des élèves & & Actions d & èves \\
\hline Superviseur $1(\mathrm{~N}=1278)$ & $74,73 \%$ & & & 25,27 & \\
\hline Superviseur $2(\mathrm{~N}=3234)$ & $70,50 \%$ & & & 29,50 & \\
\hline
\end{tabular}

Ce deuxième niveau d'analyse permet de mettre en évidence une similitude entre les deux superviseurs - l'appréhension relative des objets abordés est identique -, mais surtout quelques différences. Celles-ci se situent au niveau des catégories abordées dans les aires psychopédagogique et didactique. Le superviseur 1 se focalise sur la gestion de l'apprentissage quand le superviseur 2 l'aborde moins, mais discute en complément des aspects liés au climat et à l'espace, très peu sollicités par le superviseur 1 . Au niveau didactique, le superviseur 1 aborde plus le support didactique et le discours didactique. Le superviseur 2 insiste deux fois plus sur la préparation de leçon et commente un peu plus le choix et l'organisation des contenus.

Ce constat montre que globalement les aires du modèle centrées sur l'enseignant sont privilégiées. Si l'on se base sur les modèles de professionnalité classiques définis dans la littérature (Fuller, 1969; Katz, 1972; Huberman, 1989), lesquels soulignent l'importante centration sur soi des enseignants débutants, on peut s'attendre à ce que les futurs enseignants n'abordent pas spontanément les aires liées aux élèves. Dès lors, une analyse intéressante consiste à appréhender les opportunités laissées par les superviseurs aux futurs enseignants de s'exprimer sur les autres aires du modèle, à savoir les aires centrées sur l'élève et son apprentissage. On a déjà pu constater que les aires cognitives et d'objectivation étaient moins abordées. Néanmoins, la question soulevée exige que ces deux objets soient mis en relation avec l'initiative de la parole. Les superviseurs abordent-ils de manière proactive ces deux aires afin d'inciter les futurs enseignants à « sortir du modèle classique de professionnalité de l'enseignant débutant »? C'est à cette question, notamment, que le point suivant apporte des éléments de réponse.

\subsection{Répartition des types de prises de parole des superviseurs}

Lorsqu'ils abordent des éléments liés à l'aire didactique, les deux superviseurs ont tendance à être davantage interactifs ( $S 1=39,98 \%$ des mots prononcés et $\mathrm{S} 2=45,18 \%$ ) et proactifs $(\mathrm{S} 1=42,61 \%$ et $\mathrm{S} 2=50,78 \%$ ) que réactifs. En mettant en relation l'objet abordé et l'initiative de 
parole, le superviseur 1 aborde le discours didactique de façon à la fois interactive $\left(13,12 \%{ }^{4}\right)$ et proactive (12,30 \%), et le support didactique de façon proactive (14,62 \%). Quant au superviseur 2, la préparation de leçon est commentée en interactions (19,39 \%) et proactivement $(21,69 \%)$; le choix et l'organisation du contenu sont abordés proactivement $(11,44 \%)$.

Tableau 3 : initiative de la parole des superviseurs en fonction des objets de la pratique d'enseignement-apprentissage

\begin{tabular}{l|c|c|c} 
& Proactif & Réactif & Interactif \\
\hline & \multicolumn{3}{|c}{ Aire didactique } \\
\hline Superviseur 1 & $42,61 \%$ & $17,41 \%$ & $39,98 \%$ \\
\hline Superviseur 2 & $50,78 \%$ & $4,04 \%$ & $45,18 \%$ \\
\hline Superviseur 1 & \multicolumn{3}{|c}{ Aire psychopédagogique } \\
\hline Superviseur 2 & $43,27 \%$ & $10,3 \%$ & $46,43 \%$ \\
\hline & $44,48 \%$ & $7,19 \%$ & $48,33 \%$ \\
\hline Superviseur 1 & \multicolumn{3}{|c}{ Aire d'objectivation } \\
\hline Superviseur 2 & $60,72 \%$ & $13,15 \%$ & $26,13 \%$ \\
\hline & $64,38 \%$ & $4,54 \%$ & $31,08 \%$ \\
\hline Superviseur 1 & \multicolumn{3}{|c}{ Aire cognitive } \\
\hline Superviseur 2 & $62,18 \%$ & $8,9 \%$ & $28,92 \%$ \\
\hline
\end{tabular}

L'initiative de la parole pour l'aire psychopédagogique est marquée globalement par un relatif équilibre entre propos interactifs ( $\mathrm{S} 1=46,43 \%$ et $\mathrm{S} 2=48,33 \%)$ et proactifs $(\mathrm{S} 1=43,27 \%$ et $\mathrm{S} 2=44,48 \%)$ au détriment des propos réactifs $(\mathrm{S} 1=10,3 \%$ et $\mathrm{S} 2=7,19 \%)$. Pour les deux superviseurs, la gestion de l'apprentissage est initiée de manière proactive $(\mathrm{S} 1=27,67 \%$ et S2 = 17,93 \%) et interactive $(\mathrm{S} 1=25,42 \%$ et S2 = $17,33 \%)$. En ce qui concerne la catégorie « espace et climat », peu abordée par le superviseur 1, celui-ci ne l'aborde d'ailleurs que de manière réactive. Le superviseur 2 l'aborde de façon interactive et proactive : 9,81\% et 7,03\%.

En abordant l'initiative de la parole des deux autres aires du modèle, la centration est mise sur les opportunités offertes par le superviseur aux futurs enseignants de s'exprimer sur les aspects liés davantage aux élèves. Si ces aires sont globalement peu abordées, elles sont néanmoins majoritairement initiées par les deux superviseurs. Ainsi, l'aire d'objectivation est abordée pour le superviseur 1 à raison de $60,72 \%$ des mots exprimés par rapport à cette aire et 64,38 \% pour le superviseur 2. L’aire cognitive est caractérisée par 62,18\% pour le superviseur 1 et 59,9 \% pour le superviseur 2. Au niveau de la réactivité - durant laquelle ce sont les futurs enseignants qui abordent l'objet -, l'aire cognitive est caractérisée par un taux de 8,9\% pour le superviseur 1 et 5,84 \% pour le superviseur 2; l'aire d'objectivation : 13,15 \% pour le superviseur 1 et $4,54 \%$ pour le superviseur 2 . On peut donc en conclure, en lien avec la description des enseignants débutants proposée dans les différents modèles de la professionnalité, que les superviseurs abordent de façon proactive ces aires, mais dans des proportions qui demeurent faibles.

\subsection{Processus réflexifs mobilisés par les futurs enseignants}

Afin de compléter l'étude de la variabilité inter-superviseurs, il a semblé utile de percevoir si les deux superviseurs atteignent de la même façon l'objectif fixé, à savoir développer la réflexivité des futurs enseignants. Pour ce faire, la focale est ici mise sur les processus mobilisés par les futurs enseignants.

Les données présentées ont été obtenues à partir du nombre total de mots codés dans la catégorie " processus réflexifs ", c’est-à-dire 12927 mots pour le groupe 1 (les futurs enseignants ayant discuté avec le superviseur 1) et 11527 mots pour le groupe 2 (avec le superviseur 2 ). Les deux groupes de futurs enseignants utilisent dans des proportions assez semblables les différents processus réflexifs. Le processus réflexif le plus mobilisé est la narration (55,47\% des propos réflexifs des futurs enseignants du groupe 1 et $42,27 \%$ des propos réflexifs des futurs enseignants du groupe 2). Le deuxième processus réflexif mobilisé est la proposition d'alternatives (10,26 \% des propos réflexifs du groupe 1 et $14,62 \%$ des propos réflexifs du groupe 2). Le troisième processus réflexif le plus mobilisé est la prise de conscience dans le cas du groupe 1 (9,55 \% des propos réflexifs contre 7,86 \% des propos réflexifs du groupe 2$)$ et l'évaluation dans le cas du groupe 2 (13,53 \% des propos réflexifs contre $8,55 \%$ des propos réflexifs du groupe 1). Le processus " pointer ses difficultés » est deux fois plus présent dans les propos des futurs enseignants du groupe 2 (12,73\% de leurs propos réflexifs) que dans ceux du groupe 1 (5,90 \% de leurs propos réflexifs). Les trois types de légitimation, ainsi que les processus " intentionnaliser ", " explorer des alternatives ", " diagnostiquer ", " questionner » et " théoriser » occupent chacun moins de $5 \%$ des propos réflexifs des deux groupes.

Si les deux processus les plus représentés pour chaque superviseur sont identiques, à savoir la narration et la proposition d'alternatives, on observe tout de même certaines différences en termes de proportions des propos entre les deux groupes de futurs enseignants. De plus, la prise de conscience et l'évaluation sont, au niveau du classement, inversées, entre les deux superviseurs. Cependant, les proportions allouées à chaque processus demeurent relativement proches. Cette analyse descriptive ne permet donc pas d'identifier un « effet superviseur ».

4 Ce pourcentage signifie que 13,12\% des mots prononcés dans l'aire didactique sont de l'ordre du discours didactique abordé de manière interactive par le superviseur. 
Cet article présente une recherche permettant d'apporter des éléments de réponse à la double problématique d'objectivation et d'évaluation des traces de réflexivité des futurs enseignants et des interventions des superviseurs lors de séances de rétroaction. L'accent est ici mis sur l'analyse de la variabilité inter-superviseurs afin de s'assurer que les opportunités de développement de la réflexivité sont les mêmes pour tous les futurs enseignants, et ce, quel que soit le superviseur qui leur est attribué.

Les principaux résultats indiquent que les deux superviseurs se distinguent principalement en ce qui concerne le nombre de mots prononcés. Considérant l'efficience comme le principe visant à «diminuer les moyens alloués pour son fonctionnement, sans diminuer le degré d'atteinte des objectifs visés, c'est-à-dire sans réduire son efficacité » (Bouchard et Plante, 2002, p. 231), le superviseur 1 serait plus efficient, car il parvient à aborder, de manière générale, les mêmes objets de la pratique d'enseignement-apprentissage que le superviseur 2, tout en permettant à ses étudiants de s'exprimer autant que ceux de son collègue, et ce, en moins de temps. Nuançons toutefois ce constat, car rien n'indique que le fait de parler plus ou moins longtemps permet (ou non) aux étudiants de mieux assimiler les différents conseils qui leur sont fournis.

La transmission, bien qu'étant l'intervention la plus utilisée par les deux superviseurs, occupe une part plus importante dans les propos du superviseur 2 que dans ceux du superviseur 1. Bien qu'un des buts de la rétroaction soit de développer la réflexivité des étudiants, le superviseur ne peut pas toujours poser des questions à l'étudiant en espérant que celui-ci va se montrer réflexif. Il doit parfois " prendre la main » et répondre aux problèmes concrets que se pose le futur enseignant en adoptant un style davantage "transmissif " (Correa Molina, 2005b). En outre, le temps de formation relativement court (40 h de formation pratique avant les stages) impose aux formateurs de transmettre certains conseils aux étudiants avant leur stage, illustrant la tension entre les pôles recherche et formation à laquelle sont confrontés les superviseurs.

Si globalement les deux superviseurs abordent dans des proportions semblables les différents objets de la pratique d'enseignementapprentissage, quelques différences ont pu être observées. Celles-ci pourraient notamment s'expliquer par le fait qu'ils individualisent leurs interventions en fonction de chaque futur enseignant, de son profil, de son expérience..., "la capacité d'agir de manière contextualisée et de s'adapter aux circonstances plutôt que d'appliquer une technique ou un modèle » étant un signe de professionnalisme du superviseur (Correa Molina, 2005 b, p. 5).

Néanmoins, outre ce souci d'individualisation, il convient de s'assurer que les opportunités de développement de la réflexivité sont les mêmes pour tous les futurs enseignants, et ce, quel que soit le superviseur qui leur est attribué. L'analyse des propos des futurs enseignants en distinguant les deux groupes (futurs enseignants encadrés par le superviseur 1; futurs enseignants encadrés par le superviseur 2) indique qu'il n'existe pas de différences importantes entre ces deux groupes au niveau des processus réflexifs mobilisés. On ne peut, à ce niveau d'analyse, constater de différences au niveau des manifestations de la réflexivité qui seraient attribuables à un éventuel « effet superviseur ».

\section{Conclusion}

En Fédération Wallonie-Bruxelles, le décret de 2001 définissant la formation initiale des agrégés de l'enseignement secondaire supérieur présente les treize compétences qui doivent être développées chez les futurs enseignants. La treizième compétence est centrée sur le développement de la réflexivité. Dans le cadre de la formation pratique des agrégés de l'enseignement secondaire supérieur (pour la Faculté de Psychologie et des Sciences de l'Éducation et la Faculté Warocqué d'Économie-Gestion), l'Institut d'Administration scolaire de l'Université de Mons organise une activité visant le développement de la réflexivité : il s'agit d'un entretien de rétroaction au cours duquel le futur enseignant et un superviseur échangent au sujet de la première leçon du futur enseignant (réalisée en microenseignement) à partir de l'enregistrement vidéo de la prestation de celui-ci.

Dans le cadre de cette recherche, dix entretiens de rétroaction issus de l'année académique 2011-2012 ont fait l'objet d'une analyse de contenu thématique, réalisée à l'aide du logiciel $\mathrm{NVivo}^{\circ}$, et portant sur : (1) la nature des interventions des superviseurs et les objets de la pratique d'enseignement-apprentissage auxquels ces interventions se rapportent; (2) la proactivité, réactivité ou interactivité des interventions du superviseur; (3) les processus réflexifs mis en œuvre par les futurs enseignants.

Les résultats amènent à formuler des pistes d'amélioration de la supervision d'une part et des perspectives de recherche d'autre part. En ce qui concerne la supervision, plusieurs pistes d'amélioration peuvent être proposées. Il est important que les deux superviseurs continuent d'attirer l'attention des futurs enseignants sur les aires relatives à l'apprentissage, puisqu'ils les abordent proactivement, certes, mais dans des proportions limitées. De la même manière, les pôles « élève » et " résultat » ne sont que très peu abordés par les superviseurs. Il convient donc que les superviseurs attirent l'attention des étudiants sur ceux-ci. Étant donné que les futurs enseignants ont davantage recours au processus " proposer des alternatives " qu'au processus " explorer des alternatives », qui se distingue du premier par une évaluation de l'alternative proposée (Derobertmasure, 2012), les superviseurs pourraient encourager les futurs enseignants à évaluer les alternatives que ceux-ci proposent par des relances, des questions. Dans le même sens, les superviseurs pourraient encourager par des questions les processus de légitimation et d'intentionnalisation, peu utilisés par les futurs enseignants.

En ce qui concerne les perspectives de recherche, la façon dont l'entretien de rétroaction s'organise dans le temps pourrait également être étudiée (ex. : les consignes sont-elles toujours présentées en début d'entretien? Comment se succèdent les différents objets de la pratique 
d'enseignement-apprentissage en début, milieu et fin d'entretien?). Pour ce faire, un outil tel que le logiciel The Observer $\mathrm{XT}^{\circledR}$ pourrait être utilisé. Une première approche de ce type d'analyse de séquences de codes portant sur ces mêmes séances de rétroaction a déjà été réalisée à l'aide du logiciel QDA Miner (Derobertmasure et Robertson, 2013) dont l'une des limites techniques (analyse de séries de deux codes) ne permet pas l'analyse globale des éventuels modèles présents dans ce type de corpus. En outre, l'utilisation de la vidéo de la prestation du futur enseignant (opérations " pause ", " play ", durée de visionnement, etc.) par les deux superviseurs pourrait également être approchée par ce type d'outil.

Un autre prolongement de cette recherche serait de mener un entretien avec chaque superviseur afin de l'amener à expliquer les raisons, les intentions de ses interventions. Une démarche de ce type a été mise en place par Correa Molina (2005 b), qui a mené des entretiens semistructurés inspirés de l'approche de l'argumentation pratique (Fenstermacher, 1996) afin d'amener les superviseurs à expliciter les motifs de leurs interventions.

Enfin, les résultats obtenus dans le cadre de cette recherche permettent de fournir un retour objectif aux deux superviseurs au sujet de leur supervision. Par exemple, le superviseur 2 peut prendre conscience qu'il utilise un style davantage " transmissif » que son collègue; le superviseur 1 peut se rendre compte qu'un aspect de l'aire psychopédagogique à savoir « le climat et l'espace » est quasiment absent de ses propos. Il serait donc intéressant de travailler sur la façon de présenter les résultats aux superviseurs afin de ne pas leur fournir des données brutes de recherche.

Pour conclure, insistons sur le fait que "si celui-ci [le superviseur] endosse un rôle important, aucune préparation à cette fonction n'est mise en place. La situation de supervision est vécue, mais le superviseur ne bénéficie pas, à la suite, d’un entretien réflexif lui permettant de s'améliorer! " (Derobertmasure, 2012, p. 456). Des recherches (Dehon, Derobertmasure et Demeuse, 2014) telles que celle-ci, ainsi que les différentes perspectives proposées dans cette conclusion sont donc à encourager afin de permettre aux superviseurs d'obtenir un retour formatif sur leurs interventions.

\section{Références bibliographiques}

Bardin, L. (2001). L'analyse de contenu. Paris : Presses universitaires de France (10édition).

Beckers, J. (2009). Contribuer à la formation de "praticiens réflexifs». Pistes de réflexion. Puzzle, 26, 4-14.

Beauchamp, C. (2006). Understanding reflection in teaching : a framework for analyzing the literature. Montréal : thèse doctorale. [En ligne]. Page consultée le 15 octobre 2013. http://digitool.library.mcgill.ca/R/?func=dbin-jump-full\&object_id=100319\&local_base=GEN01MCG02

Berthier, N. (2010). Les techniques d'enquête en sciences sociales. Méthodes et exercices corrigés. Paris : Armand Colin (4e édition).

Blanchet, A. (1989). L'entretien : la coconstruction du sens. In C. Révault d’Allones (Dir.), La démarche clinique en sciences humaines (pp. 87102). Paris : Dunod.

Bocquillon, M., Derobertmasure, A., Demeuse, M., Kozlowski, D., \& Artus, F. (2014). Évaluation des enseignements par les étudiants : que nous disent les commentaires écrits des étudiants? Communication présentée dans le cadre du symposium "L'évaluation des enseignements par les étudiants en question ", 28e Congrès de l'Association internationale de Pédagogie universitaire (AIPU), Pédagogie universitaire : entre recherche et enseignement, Mons, Belgique.

Bouchard, C., \& Plante, J. (2002). La qualité : mieux la définir pour mieux la mesurer. Les Cahiers du Service de Pédagogie expérimentale, 11-12, 219-236.

Boutet, M., \& Gagné, L. (2009). Pour un dialogue réflexif entre superviseurs et supervisés. Rouen : Actes du 7e Colloque international de la Conférence des Directeurs d'IUFM (CDIUFM), Le développement professionnel des enseignants. Le point de vue des sujets.

Communauté française de Belgique. (2001). Décret définissant la formation initiale des agrégés de l'enseignement secondaire supérieur. Bruxelles : Ministère de la Communauté française. [En ligne]. Page consultée le 20 septembre 2013. http://www.gallilex.cfwb.be/ document/pdf/25595_000.pdf

Collin, S. (2010). L'interaction en ligne comme soutien à la pratique réflexive des enseignants-stagiaires. Montréal : thèse doctorale. [En ligne]. Page consultée le 4 décembre 2014. https://papyrus.bib.umontreal.ca/xmlui/handle/1866/4546

Correa Molina (2005a). Les savoirs du superviseur lors de l'entretien postobservation. In C. Gervais \& L. Portelance (Ed.), Des savoirs au coeur de la profession enseignante. Contextes de construction et modalités de partage (pp. 289-304). Sherbrooke : Editions du CRP.

Correa Molina (2005b). La mobilisation des savoirs du superviseur de stage. Université de Sherbrooke. [En ligne]. Page consultée le 30 octobre 2013. http://www.ices.fr/BU/documents/koha_99956/pdf/a4_/correa_molina_enrique.pdf

Correa Molina (2008). Les superviseurs de stage : des qualités pour un rôle formateur. In E. Correa Molina, C. Gervais, \& S. Rittershaussen (Ed.), Vers une conceptualisation de la situation de stage : explorations internationales. Sherbrooke : Editions du CRP.

Correa Molina, E., Collin, S., Chaubet, P., \& Gervais, C. (2010). Concept de réflexion : un regard critique. Éducation et francophonie, 38 (2), 135-154.

De Cock, G. (2007). Le journal de bord, support de la réflexion sur la pratique professionnelle pour les futurs enseignants en stage. Louvain-laNeuve : thèse doctorale.

Dehon, A., Derobertmasure, A., \& Demeuse, M. (2014). Appréhender l'identité professionnelle : démarches méthodologiques et implications 
épistémologiques. Construction d'un cadre de lecture et application dans un contexte de formation initiale d'enseignants. Les Dossiers des Sciences de l'Éducation, 31, 63 - 80.

De Landsheere, G., \& Bayer, E. (1974). Comment les maîtres enseignent : analyse des interactions verbales en classe. Bruxelles : Ministère de l'éducation nationale et de la culture française. Direction générale de l'organisation des études (3e édition).

De Lièvre, B. (2005). La qualité du tutorat : la complémentarité de la rigueur et de la diversité. Bejaia : Second Séminaire Euro-Méditerranéen d'Approfondissement sur la Formation à Distance. [En ligne]. Page consultée le 22 octobre 2013. http://www.google.be/url?sa=tetrct= jetq=etesrc=setsource =webetcd $=1$ etved $=0 \mathrm{CC} 0 \mathrm{QFjAAeturl}=\mathrm{http} \% 3 \mathrm{~A} \% 2 \mathrm{~F} \% 2 \mathrm{Fute} \cdot$ umh.ac.be\%2Fdeste\%2Fdownload.php\%3Ffd\%3Dre ssources\%2Fpublications\%2Fcemaforad2.pdfetei=-CNpUp7BH6qg0wWkr4HYAwetusg=AFQjCNHDzliUscfacmiVstzTL5ytffsQvAetb $\mathrm{vm}=\mathrm{bv} .55123115, \mathrm{~d} . \mathrm{d} 2 \mathrm{k}$

Derobertmasure, A. (2012). La formation initiale des enseignants et le développement de la réflexivité? Objectivation du concept et analyse des productions orales et écrites des futurs enseignants. Université de Mons : thèse doctorale. [En ligne]. Page consultée le 15 décembre 2012. http://tel.archives-ouvertes.fr/tel-00726944

Derobertmasure, A., Dehon, A., \& Demeuse, M. (2009). Évaluation d'un dispositif de formation d'enseignants. Évaluation et dévéloppement professionnel. Louvain-la-Neuve. [En ligne]. Page consultée le 30 octobre 2013. https://hal.archives-ouvertes.fr/hal-00598429/document

Derobertmasure, A., \& Dehon, A. (2012). Développement de la réflexivité et décodage de l'action : questions de méthode. Phronesis, 1 (2), 24-44.

Derobertmasure, A., \& Robertson, J.E. (2013). Data analysis in the context of teacher training : code sequence analysis using QDA MINER. Quality and quantity, 48 (4), 2255-2276.

Fallery, B., \& Rodhain, F. (2007). Quatre approches pour l'analyse de données textuelles : lexicale, linguistique, cognitive, thématique. Montréal : 16e Conférence Internationale de Management Stratégique. [En ligne]. Page consultée le 5 mai 2013. http://www.strategie-aims.com/ events/conferences/7-xvieme-conference-de-l-aims/communications/2078-quatre-approches-pour-lanalyse-de-donnees-textuelleslexicale-linguistique-cognitive-thematique/download

Fenstermacher, G. (1996). Les arguments pratiques dans la transformation morale de l'enseignement d'une discipline. Revue des sciences de l'éducation, 22 (3), 617-634.

Fuller, F. F. (1969). Concerns of teachers: a developmental conceptualization. American Educational Research Journal, 6 (2), 207-226.

Ghiglione, R., Beauvois, J.-L., Chabrol, C., \& Trognon, A. (1980). Manuel d'analyse de contenu. Paris : Armand Colin.

Hatton, N., \& Smith, D. (1995). Reflection in teacher education : towards definition and implementation. Teaching and Teacher Education, $11(1), 33-49$.

Hensler, H., Garant, C., \& Dumoulin, M.-J. (2001). La pratique réflexive, pour un cadre de référence partagée par les acteurs de la formation. Recherche et Formation, 36, 29-42.

Huberman, A.M. (1989). La vie des enseignants. Évolution et bilan d'une profession. Neuchâtel : Delachaux et Niestle.

Jorro, A. (2005). Réflexivité et auto-évaluation dans les pratiques enseignantes. Mesure et évaluation en education, 27 (2), 33-47.

Katz, L. (1972). Developmental stages of preschool teachers. Elementary School Journal, 73 (50), 123-127.

Kolb, D.A. (1984). Experiential learning : experience as the source of learning and development. Englewood Cliffs (New Jersey): Prentice-Hall.

Korthagen, F. (2001). Linking practice and theory : the pedagogy of realistic teacher education. Annual Meeting of the American Educational Research Association, Seattle.

Korthagen, F. (2011). Making teacher education relevant for practice: the pedagogy of realistic teacher education. Orbis Scholae, 6 (2), 31-50.

Korthagen, F., \& Kessels, J.P.A.M. (1999). Linking theory and practice: changing the pedagogy of teacher education. Educational Researcher, $28(4), 4-17$.

Korthagen, F., \& Vasalos, A. (2005). Levels in reflection: core reflection as a means to enhance professional growth. Teachers and Teaching: theory and practice, 11 (1), 47-71.

McAlpine, L., Weston, C., Beauchamp, J., Wiseman, C., \& Beauchamp, C. (1999). Building a metacognitive model of reflection. Higher education, 37, 105-131.

Miles, M.B., \& Huberman, A.M. (2003). Analyse des données qualitatives. Bruxelles : De Boeck (2e édition).

Quintin, J.J. (2007). Accompagnement d'une formation asynchrone en groupe restreint : modalités d'intervention et modèles idiosyncrasiques des tuteurs. Grenoble : communication au Colloque EPAL.

Saussez, F., Ewen, N. \& Girard, J. (2001). Au cœur de la pratique réflexive, la conceptualisation? Recherche et Formation, 36, 69-87.

Schön, D.A. (1994). Le praticien réflexif. À la recherche du savoir caché dans l’agir professionnel. Montréal : Les Editions Logiques.

Sparks-Langer, G., Simmons, J.M., Pasch, M., Colton, A. \& Starko, A. (1990). Reflective pedagogical thinking: how can we promote it and measure it? Journal of Teacher Education, 41 (4), 23-32.

Van Manen, M. (1977). Linking ways of knowing with ways of being practical. Curriculum Inquiry, 6, 205-228.

Zeichner, K., \& Liston, D. (1996). Reflective teaching : an introduction. Mahwah: Lawrence Erlbaum Associates 